\title{
Design of a 325MHz SC Spoke040 cavity at IHEP
}

\author{
PENG Ying-Hua(() $)^{1,2 ; 1)}$ ZHANG Xin-Yin( ()$^{1,2 ; 2)} \quad$ SHA Peng ${ }^{2}$ \\ PAN Wei-Min ${ }^{2}$ LI Han ()$^{1,2}$ \\ ${ }^{1}$ Graduate University of Chinese Academy of Science, Beijing 100049, China \\ ${ }^{2}$ Institute of High Energy Physics, Chinese Academy of Sciences, Beijing 100049, China
}

\begin{abstract}
The $325 \mathrm{MHz},=0.40$ superconducting single spoke cavity (Spoke040) was one of the most challenges for the China-ADS (Accelerator Driven System) project. The design was finished, and the fabrication was in progressing. In this paper, we studied the main radio frequency $(\mathrm{RF})$ and mechanical parameters, and compared the arc-shaped and plate-shaped end group structures..
\end{abstract}

Key words: spoke cavity, RF parameters, mechanical study

PACS: $29.20 \mathrm{Ej}$

\section{Introduction}

The successful horizontal test (H.T.) of the first $325 \mathrm{MHz},=0.12$ superconducting single spoke cavity in IHEP in September 2013 have made a milestone in the $R \& S$ of spoke cavities in China[1]. Spoke cavities operate in the fundamental TEM-mode, tested to date are designed for particle velocities $0.15<\beta<0.75[2] \mathrm{A}$ serious of $325 \mathrm{MHz},=0.40$ superconducting single spoke cavities had been designed to accelerate a $10 \mathrm{~mA}$ proton beam from $34 \mathrm{MeV}$ to $178 \mathrm{MeV}(0.26<\beta<0.54)$, next to Spoke021 cavities and then superconducting elliptical cavities in the layout of China-ADS linac 2].

With the experience of successful design, fabrication, processing and testing of the first two $325 \mathrm{MHz}$,Spoke012 in IHEP, we were more confidence in the study of SC spoke cavities. There are also many challenges in the development of Spoke040. Its mainly size was much larger than that of Spoke012 and Spoke021, which need more concern on the mechanical properties and the facture of the mold equipments. It would be the first time to study $\beta=0.40$ single SC spoke cavity in the world.

In this paper, RF design parameters and mechanical properties were respectively studied by CST microwave Studio and Ansys workbench. A new design for the end group would also been proposed.

\section{RF design}

The purpose of RF design is to get lower heat load and a higher accelerating gradient, that is get a higher $R / Q_{0}$ and lower peak surface fields (Epeak/Eacc and Bpeak/Eacc) [3]. Generally Epeak/Eacc should be slightly less than $3[4]$. Before doing the electromag- netic optimizations of the Spoke040, several parameters have been chosen in order to match China-ADS general requirements:325MHz frequency; Proton beam energy area: $34 \mathrm{MeV}-178 \mathrm{MeV}(0.26<\beta<0.54) ; \beta_{g}=0.40$; Total length $<614 \mathrm{~mm}$ (including the liquid helium bath and tuner); $\mathrm{Vcmax}=2.86 \mathrm{MV}$, come from $E a c c=V c / \beta \lambda=$ 7.7MV; Epeak $<32.5 \mathrm{MV} / \mathrm{m}$, Bpeak $<65 \mathrm{mT}$.

And we summarized the principles for designing Spoke040 cavity: Minimize the Epeak and Bpeak to match the physics requirements; Minimize the length and radius of the cavity to make it more tight to use the already existing V.T. (vertical test) facilities and the end flanges; Simple the structure, make it easier to fabrication and progress; $\mathrm{R} / \mathrm{Q}$ and $\mathrm{G}$ should be as large as possible to achieve promising $Q_{0}$, decrease the $P_{c}$.

\subsection{The sensitive analyze}
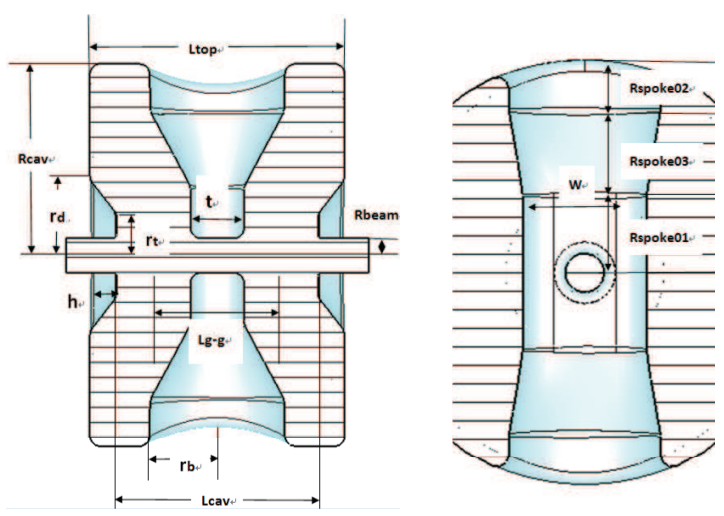

Fig. 1. Cut-away views of the spoke cavity model in the MWS and the main geometric parameters

Received 14 March 2009

1) E-mail: pengyh@mail.ihep.ac.cn

2) E-mail: zhangxy@mail.ihep.ac.cn 
We used the original design progress to choose the main geometric parameters for the Spoke 040 cavity [4, 5]. First we chosen $L g-g=\beta_{g} \lambda / 2=185.0 \mathrm{~mm}, L \mathrm{cav}=$ $2 / 3 *{ }_{g} \lambda=247.0 \mathrm{~mm}, 2 * \mathrm{rb} /$ Lcav $=0.4, \mathrm{~W} /$ Lcav $=$ 0.8 andRspoke $01=$ Rspoke $02=$ Rspoke 03 . Then sweeping the main geometric parameters around those values to analysis how sensitive the RF parameters to the changes would be. The results were showed in Fig2.

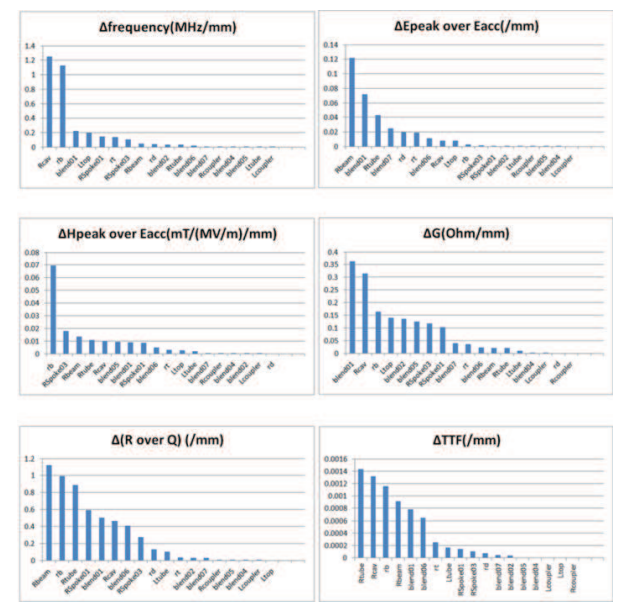

Fig. 2. The RF parameters change sensitive to geometric value

Taking $r_{d}$ (end wall bottom radius) as an example:when $\Delta r_{d}=1 m m, \Delta f=1.1 M H z, \Delta($ Epeak $/$ Eacc $)=$ $0.02, \Delta($ Hpeak $/$ Eacc $)=(0.07 m T / M V) / m, \Delta(R / Q)=$ $1, \Delta G=0.15 \mathrm{Ohm}, \Delta T T F=0.001$. The results showed the $r_{d}$ mostly influence $H_{p} e a k / E_{a} c c$, fandR/Q; had less influence on TTF and G; and almost no influence on $E_{p} e a k / E_{a} c c$.These provided us an effective criterion for more details RF design.

\subsection{RF optimization of the plate-shaped and arc-shaped end groups}

The Spoke040 cavitys main sizes are much larger than that of Spoke021 and Spoke012 cavities. Considering improving the mechanical performance, we compared two types of end group to find a better geometric combination. Fig3 showed the difference between the end groups. Table1 showed the main geometric chose and table2 showed the RF parameters.
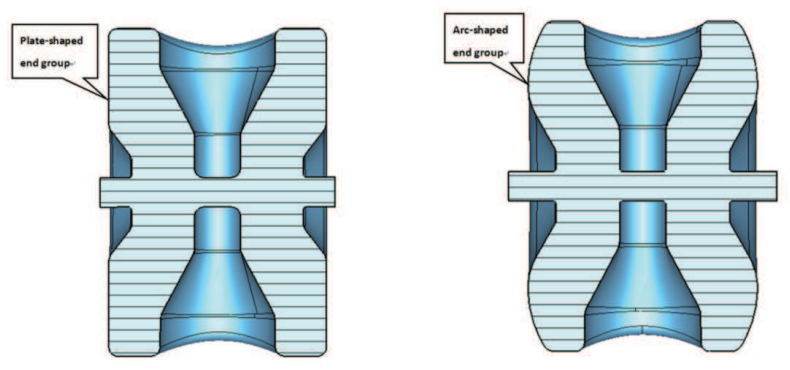

Fig. 3. Cut-away view of the plate-shaped (left) and arc-shaped (right) end groups

Table 1. Geometric parameters of the two types end group of Spoke040

\begin{tabular}{lcccc}
\hline & Ltop $/ \mathrm{mm}$ & Lcav $/ \mathrm{mm}$ & Rcav $/ \mathrm{mm}$ & $W / \mathrm{mm}$ \\
\hline Plate-shaped & 370 & 292 & 278 & 160 \\
Arc-shaped & 386.6 & 292 & 278 & 160 \\
\hline \hline & Rbeam $/ \mathrm{mm}$ & $r_{d} / \mathrm{mm}$ & $r_{t} / \mathrm{mm}$ & $r_{b} / \mathrm{mm}$ \\
\hline Plate-shaped & 25 & 110 & 60 & 98 \\
Arc-shaped & 25 & 120 & 60 & 98 \\
\hline
\end{tabular}

Table 2. Main RF parameters of the two types end group of Spoke040

\begin{tabular}{cccc}
\hline RFparameters & Units & Plate-shaped & Arc-shaped \\
\hline $\mathrm{f}$ & $\mathrm{MHz}$ & 324.41 & 324.44 \\
Epeak/Eacc & $\mathrm{M}$ & 2.82 & 3.68 \\
Hpeak/Eacc & $\mathrm{mT} /(\mathrm{MV} / \mathrm{m})$ & 6.25 & 8.31 \\
$\mathrm{Q}$ & & 22923.0 & 22795.6 \\
$\mathrm{R} / \mathrm{Q}$ & & 247.22 & 250.41 \\
$\mathrm{TTF}$ & & 0.817 & 0.821 \\
\hline
\end{tabular}

Compare to the plate-shaped end group, we just increased the Ltop and $r_{d}$ of the arc-shaped end group to compensate the wave transverse volume to adjust the frequency on the basis of microwave perturbation theory. The main influence on RF parameters were the increases of Epeak/Eacc from 2.82 to 3.68 and Hpeak/Eacc from 6.25 to $8.31 \mathrm{mT} /(\mathrm{MV} / \mathrm{m})$.

\section{Mechanical studies}

We simulated the naked shells mechanical parameters in the room temperature $T=295.3 K$ with niobium in different thickness and pressure while the beam ports were locked or not. The material properties of the niobium used are: Density $8600 \mathrm{~kg} / \mathrm{m}^{3}$, Youngs modulus $1.03 e 11 P a$, Poisson ratio 0.38 . The results were shown in Fig4 and Table3. 


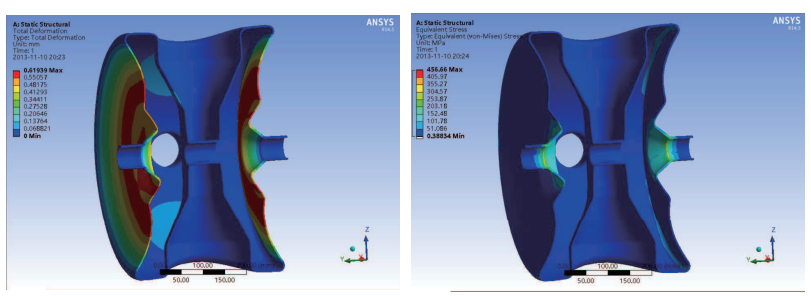

Fig. 4. Simulation of deformation (left) and stress (right) for Spoke040 @ the end group was plateshaped, $3.0 \mathrm{~mm} \mathrm{Nb}$ shell, 1atm and free beam ports.

Table 3. Some mechanical results for naked Spoke040 of different shapes, thickness, and pressure @ locked beam ports

\begin{tabular}{cccc}
\hline \multicolumn{4}{c}{ Plate-shaped } \\
\hline Peak stress./MPa & $3.0 \mathrm{~mm}$ & $3.5 \mathrm{~mm}$ & $4.0 \mathrm{~mm}$ \\
\hline $1.0 \mathrm{~atm}$ & 556.56 & 347.88 & 448.58 \\
$1.5 \mathrm{~atm}$ & 834.85 & 521.81 & 672.86 \\
$2.0 \mathrm{~atm}$ & 1113.1 & 695.75 & 897.15 \\
\hline Deformation./mm & $3.0 \mathrm{~mm}$ & $3.5 \mathrm{~mm}$ & $4.0 \mathrm{~mm}$ \\
\hline $1.0 \mathrm{~atm}$ & 0.46617 & 0.46611 & 0.37654 \\
$1.5 \mathrm{~atm}$ & 0.96476 & 0.69916 & 0.56481 \\
$2.0 \mathrm{~atm}$ & 1.2863 & 0.93221 & 0.75308 \\
\hline \hline & Arc-shaped & & \\
\hline Peak stress./MPa & $3.0 \mathrm{~mm}$ & $3.5 \mathrm{~mm}$ & $4.0 \mathrm{~mm}$ \\
\hline $1.0 \mathrm{~atm}$ & 309 & 362.2 & 389.48 \\
$1.5 \mathrm{~atm}$ & 463.5 & 543.3 & 584.22 \\
$2.0 \mathrm{~atm}$ & 618 & 724.39 & 778.96 \\
\hline Deformation./mm & $3.0 \mathrm{~mm}$ & $3.5 \mathrm{~mm}$ & $4.0 \mathrm{~mm}$ \\
\hline $1.0 \mathrm{~atm}$ & 0.42168 & 0.33315 & 0.26586 \\
$1.5 \mathrm{~atm}$ & 0.63251 & 0.49972 & 0.39879 \\
$2.0 \mathrm{~atm}$ & 0.84335 & 0.66629 & 0.53172 \\
\hline
\end{tabular}

The results shown the total deformation was mainly found symmetrically around the beam tube. And the max deformation appeared at the joint line of the arc and the slope, where the ring added should function to make the compromise. The press mainly gathered at the bottom of the beam tube symmetrically. The deformation and press decreased while the $\mathrm{Nb}$ shells thickness increased, but the material extensibility and hardness should also been concerned in the fabrication. Take the $3.0 \mathrm{~mm}$, 1atm situation as an example: the deformation and press of arc-shaped end group were much smaller than those of plate-shaped end group, which made us believe the sacrifice of the RF parameter in choosing the arc-shaped was worthy.

\section{Conclusion}

The study of the $325 \mathrm{MHz}, \beta=0.40$ superconducting single spoke cavities was one of the key challenges for the China-ADS main linac, and also no precedent in the world. In this paper we introduced the optimization of the main geometric parameters, the studies of RF and naked mechanical parameters, and the compare of arc-shaped to plate-shaped end groups. The first SC Spoke040 cavity was just under construction. More works about the analysis of multipacting, the design of the rings and the study of the tuning sensitivity would be published. The work for the processing and the test were also in preparing.

\section{References}

1 http://acc1.ihep.ac.cn/tzgg/2013n/09/424210.shtml

2 Shepard K W, Ostromov P N, Delayen J R. Phys. Rev. ST Accel. Beams, 2003,6:080101

3 LI Han, SHA Peng, DAI Jianping, HUANG Hong, WANG Qunyao. Design study on very low Beta spoke cavity for ChinaADS. Chinese Physics .C(HEP\&NP),2012,36(8):761-764
4 Kelly M. Status of Superconducting Spoke Cavity Development. Proceedings of 13th International Workshop on RF Superconductivity. China:Peking University,2007

5 YAO Zhong-Yuan, LU Xiang-Yang, ZHAO Kui. Design of a $450 \mathrm{MHz},=0.20$ single spoke cavity at PKU. Chinese Physics .C(HEP\&NP), 2009,33(4):292-296

6 ZHU Feng, QUAN Sheng-Wen, WANG Li-Fang, ZHAO Kui, CHEN Jia-Er. Design of a $=0.45, \mathrm{f}=350 \mathrm{MHz}$ spoke cavity. HEP\&NP,2005,29(1):77-80 